\title{
ALEITAMENTO MATERNO EXCLUSIVO: UM ATO DE AMOR E DE EXTENSÃO!
}

\author{
FARO, S. M. de L. ${ }^{1}$, SILVA, M. J. F. ${ }^{2}$, GONÇALVES, B. H. ${ }^{3}$, SILVA, A.B.F. ${ }^{4} \&$ VALLINOTO, I. M.V. C. ${ }^{5}$ \\ ${ }^{1}$ Graduanda pela Universidade Federal do Pará (UFPA). E-mail: sarahmfaro@ gmail.com. ${ }^{2}$ Médico Graduado pela Universidade Federal do \\ Pará (UFPA). E-mail: marcelloufpa@gmail.com. ${ }^{3}$ Graduanda pela Universidade Federal do Pará (UFPA). E-mail: \\ gholandabeatriz@gmail.com. 4Médica graduada pela Universidade Federal do Pará (UFPA). E-mail: abfavacho@ gmail.com. 5Docente da \\ Universidade Federal do Pará (UFPA) e orientadora da ação extensionista de mesmo nome. E-mail: ivallinoto@ufpa.br. \\ DOI: https://doi.org/10.32356/exta.v2.n18.39759 - Artigo submetido em 28/11/2018
}

\section{RESUMO}

O aleitamento materno é considerado um dos pilares fundamentais para a promoção e para a proteção da saúde das crianças em todo o mundo. Apesar disso, estudos apresentam estatísticas relacionadas à baixa adesão ao aleitamento materno exclusivo (AME). Capacitar estudantes de medicina e de nutrição a informarem gestantes e lactantes, sobre a importância do AME, além de doar potes de leite para o Banco de Leite Humano (BLH). Participaram da capacitação, ministrada por nutricionista, alunos de medicina e de nutrição. As ações ocorreram em unidades de saúde, os voluntários conversaram com as mães sobre os temas e fizeram cadastro das que quisessem ser doadoras de leite. Também foi feita uma gincana com alunos de segundo a quinto anos de escola de ensino fundamental para arrecadar potes de vidro. Para divulgação do projeto, foi criado um perfil em rede social. As atividades nas unidades de saúde conseguiram 30 novos cadastros ao BLH, mais de 120 potes foram recolhidos e a campanha online obteve 650 seguidores em seu perfil. A capacitação e a atuação dos universitários nas unidades de saúde proporcionou-lhes importante aprendizado. Também, informar todas as gestantes sobre os benefícios e o manejo do aleitamento materno é necessário. O projeto AME mostrou-se exitoso, mesmo com as limitações no método. Dessa forma, os autores consideram o projeto AME importante e sugerem sua replicação em demais realidades.

PALAVRAS-CHAVE: Aleitamento Materno; Banco de Leite Humano; Educação em Saúde.

\section{EXCLUSIVE MATERNAL BREASTFEEDING: AN ACT OF LOVE AND EXTENSION!}

\begin{abstract}
The breastfeeding is considered one of the fundamental pillars to promotion and protection of the health child around the world; but studies show low adherence to exclusive breastfeeding. To empower the medicine and nutrition students to better inform pregnant and lactation mothers about the importance of exclusive breastfeeding, as well as on the milk donation to the Human Milk Bank. Medicine and nutrition students participated in the training, taught by nutritionist. The actions took place in health facilities, where the volunteers talked with their mothers about the themes and registered those who manifested wish of being milk donors. A gymkhana was made with second to fifth year students of elementary school to
\end{abstract}

raise glass jar. For Project disclosure, was created a profile on social network. The activities on health units got 30 new registers on human milk bank, more than 120 jars were collected and the online campaign got 650 followers in its profile. The empowerment and the college students acting on health units provided them important knowledge. Moreover, to inform all the pregnant about the benefits and the management to breastfeeding is necessary. The Exclusive Breastfeeding Project proved successful, even with the method limitations. Thus, the authors considered important the Project and suggest their replication in other realities.

KEYWORDS: Breastfeeding; Milk Bank; Health Education.

INTRODUÇÃO 
O aleitamento materno é considerado um dos pilares fundamentais para a promoção e para a proteção da saúde das crianças em todo o mundo. A amamentação no seio materno deve começar cedo, ser exclusiva até os 6 meses de idade e ser mantida como complemento alimentar nos 2 primeiros anos de vida da criança, devido aos seus benefícios nutricionais, imunológicos, cognitivos e sociais. Apesar disso, estudos apresentam estatísticas preocupantes relacionadas à baixa adesão ao aleitamento materno, especialmente, o exclusivo, mesmo sendo uma recomendação da Organização Mundial de Saúde (OMS) (VICTORA, 2016).

O aleitamento materno não é importante apenas por ser um alimento rico em nutrientes que a criança precisa, mas, também, por ser rico em anticorpos, protegendo a criança de diversas doenças, como diarreia, infecções respiratórias, alergias. Também diminui o risco de hipertensão, colesterol alto, diabetes e obesidade (BRASIL, 2018). A OMS e o Fundo das Nações Unidas para Infância (Unicef) mostram que cerca de seis milhões de crianças estão sendo salvas da mortalidade infantil, por ano, por conta do aumento das taxas de amamentação exclusiva, principalmente as de menor nível socioeconômico, as quais possuem maior risco de morrer no primeiro ano de vida. Nenhuma outra estratégia isolada possui a mesma eficácia na redução das mortes de crianças menores de cinco anos (BRASIL, 2009).

A amamentação estimula uma ligação entre mãe e filho, por propiciar um contato mais profundo. $\mathrm{O}$ ato da sucção, também, ajuda no desenvolvimento da face, da fala e de boa respiração na criança, por propiciar uma melhor conformação do palato duro, o qual é fundamental para o alinhamento correto dos dentes e da boa oclusão dentária. Evidências também mostram que o aleitamento materno contribui para o desenvolvimento cognitivo (BRASIL, 2009). Fonseca et al (2013) mostraram que componentes lipídicos importantes para o desenvolvimento das membranas celulares, principalmente das células do sistema nervoso central, as quais rapidamente se mielinizam nos primeiros dois anos de vida, estão presentes no leite materno.

As ações sobre a importância do aleitamento são essenciais, visto que as mães e os familiares ainda possuem muitas dúvidas. É comum ouvirmos mães ou acompanhantes falarem que o "leite é fraco" por conta do choro infantil, quando, muitas vezes, são pequenos detalhes que precisam ser ajustados na pega ou então o choro não tem a ver com a alimentação da criança. O Caderno da Atenção Básica de 2009 mostra que a promoção da amamentação na gestação possui um impacto positivo na prevalência do aleitamento materno, principalmente entre as primíparas. A sensibilização da importância do aleitamento já deve ser feito nas 
consultas de pré-natal. Ademais, a promoção de atividades de educação em saúde sobre o aleitamento materno, ajuda na difusão do conhecimento tanto para a família da criança como para toda a comunidade no qual o profissional está inserido.

Dessa forma, o trabalho visou capacitar estudantes de medicina e de nutrição para que estejam aptos a desenvolverem as atividades do Projeto de Aleitamento Materno Exclusivo (AME), organizado por estudantes de medicina da Universidade Federal do Pará (UFPA). Ademais, o projeto também teve como fim compartilhar informações para as gestantes e lactantes sobre a importância e os benefícios do Aleitamento Materno Exclusivo (AME), além de ensinar sobre a técnica correta da pega ao seio e sobre as propriedades do leite materno, por meio de atividades desenvolvidas em unidades de atenção primária e secundária à saúde. Além disso, as ações desenvolvidas, também, tiveram como intuito a doação de potes de leite para o Banco de Leite Humano (BLH) da Fundação Santa Casa de Misericórdia do Pará (FSCMP) para o uso em neonatos e em lactentes que não podem ser amamentados com o leite das mães devido a alguma contra indicação. Também foi objetivo do projeto a sensibilização dos alunos de ensino fundamental sobre a temática em questão e sobre verminoses, com intuito de que se tornassem disseminadores do que aprenderam.

\section{MATERIAIS E MÉTODOS}

O tipo de estudo desenvolvido foi transversal, descritivo e qualitativo. O ambiente de realização do projeto englobou a Faculdade de Medicina da UFPA (FAMED UFPA), a Unidade Básica de Saúde (UBS) do Guamá, a Unidade de Referência Materno Infantil e do Adolescente (UREMIA), a Escola Estadual de Ensino Fundamental Rui Barbosa (EEEFRB) e a Fundação Santa Casa de Misericórdia do Pará (FSCMP). A divulgação do projeto foi realizada por meio do Instagram®, da IFMSA Brazil UFPA, parceira do projeto, e do Projeto AME, sendo o último um perfil criado exclusivamente para divulgação da ação.

A população englobada no projeto foram os estudantes de medicina e de nutrição que participaram do projeto, as grávidas e lactantes abordadas durante a ação, os alunos de ensino fundamental que participaram da dinâmica e os seguidores do perfil criado no Instagram®. Os públicos alvos da atividade foram as grávidas e lactantes.

O projeto ocorreu entre trinta de julho de 2015 até trinta e um de agosto de 2015 e contou com as seguintes etapas: reunião de organização do projeto, divulgação deste em rede social, capacitação dos estudantes sobre a temática, sensibilização das mulheres sobre AME e doação 
de leite materno em unidades de saúde, gincana para arrecadação de potes de leite em escola de ensino fundamental e doação dos potes arrecadados para o banco de leite da FSCMP. Os recursos utilizados foram os dos idealizadores do projeto. Os materiais adquiridos foram para a realização da atividade na escola, sendo eles cartazes de quatro cores, giz, dado, papel cartão, balões, papéis e impressão nos papéis.

Não houve método padrão de avaliação de impacto do projeto, sendo a avaliação realizada por meio dos números brutos conseguidos por número de potes de leite arrecadados, pelo número de novas doadoras cadastradas no BLH e pelo número de seguidores na rede social.

\section{RESULTADOS}

Após levantamento bibliográfico sobre o tema, cinco alunos de medicina filiados à Federação Internacional das Associações dos Estudantes de Medicina do Brasil Comitê Local da UFPA (IFMSA Brazil UFPA), coordenadores da atividade, reuniram-se com a professora e orientadora do projeto na Faculdade de Medicina (FAMED) da UFPA, no dia trinta de junho de 2015, a fim de elaborarem, conjuntamente, a atividade. Na reunião, decidiu-se que o Projeto AME ocorreria em etapas, sendo elas, capacitação dos estudantes; informação da população sobre amamentação em unidades de saúde; gincana em escola para arrecadação de potes de leite e entrega destes ao BLH.

Em seguida, houve divulgação do projeto na rede social, Instagram®, da IFMSA Brazil UFPA e do Projeto AME, sendo o último um perfil criado exclusivamente para divulgação da ação. A arte postada continha um convite aos estudantes de medicina e de nutrição a participarem da capacitação teórico-prática sobre o tema, assim como das demais atividades idealizadas. As datas e os locais de cada uma das ações estavam explícitos na arte. Havia, também, o pedido de doação de potes de vidro com tampa de plástico, assim como os contatos pelos quais as pessoas poderiam falar com coordenadores da ação para que os mesmos fossem buscar os potes. A arrecadação, também, ocorreu na escola em que o projeto foi realizado, por meio da doação dos alunos como parte da gincana. O perfil do projeto AME recebeu 650 seguidores em duas semanas e teve um alcance tão grande que os coordenadores foram convidados a divulgar a ação em um programa de televisão local.

Participaram da capacitação quatorze alunos de medicina da UFPA e seis de nutrição, sendo os últimos vindos de três instituições de ensino superior presentes em Belém do Pará. Ela aconteceu dia trinta de julho de 2015 na FAMED UFPA e foi ministrada por uma nutricionista. 
Ela instruiu os alunos sobre a importância da amamentação materna exclusiva durante os seis primeiros meses de vida da criança, informou acerca dos tipos de aleitamento materno, acerca da duração da amamentação, sobre a produção de leite, suas características e funções e, também, acerca da técnica adequada de amamentação. Além disso, falou-se sobre os aconselhamentos que devem ser dados para as mães desde o pré-natal até o retorno dela para o trabalho, como métodos de ordenha e de conservação do leite. Indicações e contra indicações do aleitamento foram faladas. Também foi frisado o manejo de situações especiais e eventuais problemas relacionados à amamentação, como mastite, por exemplo.

Nos dias três, quatro, cinco e seis de agosto de 2015 foram realizadas as ações de sensibilização na Unidade Básica de Saúde (UBS) do Guamá e na Unidade de Referência Materno Infantil e do Adolescente (UREMIA). Previamente aos dias das ações, ocorreu uma reunião com cada diretor de cada unidade a fim de explicar o intuito da atividade e obter aval para sua realização. Nesses dias, os acadêmicos abordaram grávidas e mães que estavam presentes nas unidades, de maneira individual, onde um acadêmico falava com uma mãe. Os temas abordados foram os mesmos da capacitação, mas com linguagem mais clara e objetiva, focando na importância do AME e ressaltando a importância da doação de leite materno para os centros de arrecadação de leite. Houve um momento de esclarecimento das dúvidas das mulheres. Ao final da conversa, foi feito o cadastro das mulheres que se dispuseram a serem doadoras de leite para o banco de leite da FSCMP. Tal atividade sensibilizou gestantes e lactantes.

A próxima etapa do projeto foi realizada na Escola Estadual de Ensino Fundamental Rui Barbosa (EEEFRB), tendo ocorrido o aval prévio da diretora para realização da ação. No dia sete de agosto de 2015, os coordenadores foram à escola e informaram, de turma em turma, que no dia vinte e um de agosto, dia da ação, haveria uma competição entre as salas. Participariam da competição os alunos dos segundo ao quinto anos do ensino fundamental. Nela, seriam contabilizados pontos tanto da gincana a ser realizada quanto da pontuação referente à quantidade de potes de armazenamento de leite arrecadados por cada turma.

No dia marcado, os alunos de ensino fundamental foram divididos por turma, contabilizando quatro equipes no total. A gincana aconteceu na quadra da escola, a qual foi dividida em quatro estações nas quais havia rodízio entre as equipes, sendo que cada uma correspondia a uma cor, sendo elas azul, rosa, vermelho e verde. A primeira parte da gincana começou com explicação para todas as equipes acerca de verminoses, tanto sobre agentes 
etiológicos, como métodos de transmissão, sintomas, tratamento e prevenção, explicados por um dos universitários, de maneira direcionada para as crianças, usando linguajar adequado. Em seguida, os alunos foram divididos nas suas determinadas equipes e cada uma recebeu um cartaz ilustrado com imagens representativas das doenças e cada criança falava sobre o que se lembrava da explicação e completava abaixo. A equipe que mais se lembrou da explicação, ganhou um ponto.

A segunda dinâmica foi a do tabuleiro de giz desenhado no chão. Cada equipe escolheu um representante e este jogava um dado para saber quantas casas iria avançar. Chegando à casa respectiva ao número do dado, o monitor lia uma frase do cartão a qual continha incentivo para que as crianças realizassem prevenção adequada das doenças, como "Parabéns, você sempre lava as mãos antes das refeições". "Avance uma casa" e "Eita! Um verme na sua barriguinha está lhe causando enjoos e perda de peso. Volte duas casas". Pontuava na atividade o representante da equipe que conseguisse chegar, por primeiro, no décimo quadrado do tabuleiro.

Outra dinâmica foi a das frases escondidas no balão. Todos os alunos foram divididos em suas respectivas equipes e os alunos de cada equipe foram colocados em fila indiana em um lado da quadra enquanto, do lado aposto, havia balões no chão. Em cada um havia uma frase diferente, relacionadas a bons e a maus hábitos de higiene. Quando o monitor dava o comando, o primeiro da fila corria para buscar um balão e corria de volta para entregar o balão para ser estourado na frente do monitor, o qual lia a fase e pontuava as que fossem relacionadas a bons hábitos. A equipe que pontuava era a que conseguisse chegar a cinco frases de bons hábitos primeiro que as outras.

A quarta atividade realizada foi o jogo da memória, no qual um representante de cada equipe deveria relacionar cada figura representativa de algum sintoma à carta em que estava a forma de prevenção da doença. Por exemplo, a carta de "náusea" se relacionava com a carta de "lavar bem os alimentos", pois era representativa de verminoses. Pontuava a equipe que conseguisse acertar mais pares. Ao final das estações, foram contabilizados os pontos de cada equipe na gincana, juntamente com os pontos referentes à quantidade de potes de viro arrecadados, sendo que cada pote correspondia a um ponto. A equipe vencedora foi a que obteve mais pontos. No que tange à participação do projeto na escola de ensino fundamental, o projeto teve grande valia à medida que promoveu educação em saúde, de forma lúdica e interativa, a respeito das doenças comuns da infância, contribuindo para o crescimento saudável destas. 
A última etapa do projeto foi no dia 31 de agosto de 2015 com a entrega dos potes de leite, arrecadados tanto na gincana na escola como pelos coordenadores e participantes da ação na faculdade, para o BLH da FSCMP, ajudando, assim, a maternidade e várias famílias.

Quanto às doações de leite e de potes de vidro, o êxito é notado nos mais de 30 novos cadastros no banco de doações e nos mais de 120 potes recolhidos, dentre gincana na escola e arrecadação pela divulgação em geral. Neste sentido, a campanha online foi muito abrangente e teve grande participação, revelando que a utilização das redes sociais neste tipo de projeto traz grande benefício, pois é capaz de abranger um público diverso e amplo, que talvez não fosse alcançado sem tal recurso. Sua participação expressiva pôde ser notada na quantidade de seguidores em seu perfil no Instagram ${ }^{\circledR}$.

Quanto aos acadêmicos dos cursos de medicina e de nutrição participantes, o Projeto AME atuou como amplo cenário de prática clínica, sobretudo no que diz respeito à construção da relação médico-paciente, tendo em vista que propiciou a necessidade de comunicação com públicos muito diversos, desde gestantes e puérperas, até à faixa etária pediátrica, revelando-se como boa oportunidade de promoção da educação profissional em saúde. Além disso, também fomentou as habilidades de comunicação com a comunidade, tanto de forma direta para arrecadação dos potes de leite, quanto pela gestão de marketing em redes sociais para divulgação do projeto bem como a articulação com gestores de sistemas de saúde nos diversos níveis hierárquicos.

\section{DISCUSSÃO}

A capacitação e a atuação dos universitários nas unidades de saúde, locais de prática clínica das universidades, e o contato maior com a comunidade que atendem, por meio da abordagem individual às mulheres, proporcionou-lhes importante aprendizado. É o que Costa e Silva et al (2011) corroboram, ao afirmarem que atividades de extensão possibilitam a articulação dos conteúdos, criando oportunidades para desenvolvimento de habilidades de trabalho em equipe, além de desenvolver e fortalecer o compromisso social e ético. Além disso, a extensão universitária, também, visa à socialização de conhecimentos obtidos em sala de aula com a comunidade, assim como o aprofundamento de conhecimentos teóricos sobre uma determinada área profissional (COSTA E SILVA, 2011; SANTOS, 2012).

Também é importante lembrar que uns dos Dez Passos para o sucesso no Aleitamento Materno é informar todas as gestantes sobre os benefícios e o manejo do aleitamento materno 
e mostrar às mães como amamentar e como manter a lactação, mesmo se vierem a ser separadas dos filhos (SILVA et al, 2017; BRASIL, 2017). Pereira et al (2010) mostraram, em seu estudo sobre fatores associados ao AME na atenção básica, que as ações de promoção, de proteção e de apoio à amamentação que se mostraram associadas ao aleitamento materno exclusivo foram: ter sido falado sobre amamentação em grupo na unidade de saúde que a mãe frequenta e ter recebido alguma orientação sobre amamentação, como mostrar a técnica correta de amamentação. Tais ações foram abordadas no projeto, principalmente com as lactantes que estavam com seus filhos na hora da abordagem e, com as grávidas fez-se a orientação cabível.

Vale ressaltar que, segundo Galvão et al (2006), em sua pesquisa sobre os motivos de doação de leite das mulheres cadastradas no BLH de uma maternidade pública em Fortaleza$\mathrm{CE}$, a maioria das nutrizes só tomaram conhecimento da existência de BLH no período prénatal ou no pós parto, sendo que a maioria começou a doar não necessariamente pelos objetivos inerentes dos bancos de leite, pois elas não tinham essa informação, mas sim por conta de ingurgitamento mamário. No trabalho de Neves et al (2011) com puérperas internadas no alojamento conjunto, gestantes do pré-natal e doadoras cadastradas no BLH, mesmo 52\% das mulheres entrevistadas conhecendo o BLH, $61 \%$ não conheciam, de fato, o trabalho prestado e $21 \%$ não doariam leite por falta de informação. As participantes deram como sugestões para aumentar o alcance das informações do BLH a maior divulgação dos serviços prestados e também falar mais sobre o assunto nas UBS. Dessa forma, pode-se perceber que as ações realizadas pelo projeto AME estão em consonância com as expectativas das mulheres e se mostram necessárias para o aumento da divulgação dos objetivos do BLH.

Em relação aos alunos de ensino fundamental, o trabalho de Fujimori et al (2008) mostrou que, mesmo que $96 \%$ dos alunos que pretendem tem filhos querem que eles sejam amamentados, apenas 29,3\% sabiam que o leite humano contém todos os nutrientes que os bebês precisam. Tal disparidade corrobora com a ideia de tentar ensinar desde cedo as crianças sobre os benefícios do leite materno. Além disso, informar sobre verminoses e seus métodos de contágio e de prevenção também é bastante importante, como relatado por Boeira et al (2010) em seu estudo com crianças de ensino fundamental, no qual a taxa de infecção antes das atividades lúdicas era de 36,8\% e, após as atividades, tiveram nível de reinfecção de 13,04\%, mostrando a importância do esclarecimento de informações para crianças. 


\section{CONSIDERAÇÕES FINAIS}

O projeto AME mostrou-se exitoso no que se propôs a fazer, visto que capacitou os universitários a disseminarem informações sobre o tema com gestantes e lactantes. Tal abordagem se mostrou eficaz, pois, elucidou a importância desse ato não apenas como altruísmo, mas como algo benéfico à saúde da mãe e das crianças, resultando em novas doadoras de leite cadastradas ao BLH, como também, o alcance da divulgação do projeto ao proporcionar a doação de potes de leite para a maternidade. Ademais, a abordagem lúdica da gincana caracterizou-se como um método de promoção da saúde ao informar sobre verminoses comuns da infância, contribuindo para o crescimento saudável das crianças, além de sensibilizar sobre o aleitamento materno exclusivo e a doação de leite, por meio da competição.

Além disso, vale ressaltar que houve limitações no método, como a não documentação da quantidade exata de mulheres abordadas pelos participantes nos dias de realização da ação, assim como a falta de documentação da quantidade exata de crianças que participaram da gincana. Também é importante frisar a falta de um método formal de avaliação de impacto da atividade, tanto em relação aos universitários que participaram, quanto em relação às mulheres abordadas. Vale lembrar que não foi realizada uma capacitação prévia dos universitários sobre os temas tratados na gincana, visto que se considerou que eles possuíssem tal conhecimento prévio. Dessa forma, os autores consideram o projeto AME importante e sugerem sua replicação em demais realidades, com as adaptações pertinentes realizadas.

\section{REFERÊNCIAS}

BRASIL. Ministério da Saúde. Secretaria de Atenção à Saúde. Departamento de Atenção Básica. Saúde da criança: nutrição infantil: aleitamento materno e alimentação complementar. Brasília: Editora do Ministério da Saúde, 2009. Disponível em: <http://bvsms.saude.gov.br/bvs/publicacoes/saude_crianca_nutricao_aleitamento_alimentacao .pdf>. Acesso em: $30 \mathrm{dez} .2019$.

BRASIL. Ministério da Saúde. Secretaria de Atenção à Saúde. Departamento de Ações Programáticas Estratégicas. Bases para a discussão da Política Nacional de Promoção, Proteção e Apoio ao Aleitamento Materno. Brasília, Ministério da Saúde, 2017. Disponível em:

<http://bvsms.saude.gov.br/bvs/publicacoes/bases_discussao_politica_aleitamento_materno.p df>. Acesso em: 30 dez. 2019.

BRASIL. Ministério da Saúde Secretaria de Atenção à Saúde. Departamento de Atenção Básica. Saúde da criança: aleitamento materno. Brasília: MS, 2018. Disponível em: 
<http://portalms.saude.gov.br/saude-para-voce/saude-da-crianca/aleitamento-materno>. Acesso em: 27 nov. 2018.

BOEIRA, V. L.; GONÇALVES, P. A. R. R.; MORAIS, F. G.; SCHAEDLER, V. M. Educação em saúde como instrumento de controle de parasitoses intestinais em crianças. Revista Varia Scientia, v.09, n.15, p. 35-43, 2010. Disponível em: <http://erevista.unioeste.br/index.php/variascientia/article/view/3917/3032>. Acesso em: 30 dez.2019.

COSTA, A.; SILVA, P. B. Extensão universitária brasileira: possibilidades, limitações e desafios. São Paulo: Nelpa, 2011

FONSECA, A. L. M.; ALBERNAZ, E. P.; KAUFMANN, C. C.; NEVES, I. H.; FIGUEIREDO, V. L. M. Impacto do aleitamento materno no coeficiente de inteligência de crianças de oito anos de idade. J. Pediatr. (Rio J.), Porto Alegre, v. 89, n. 4, p. 346-353, aug. 2013. Disponível em: <http://www.scielo.br/scielo.php?script=sci_arttext\&pid=S0021$75572013000400005 \& \operatorname{lng}=e n \& n r m=i s o ~>$. Acesso em: 30 dez. 2019.

FUJIMORI, M.; MORAIS, T. C.; FRANÇA, E. L.; DE TOLEDO, O. R.; HONÓRIOFRANÇA, A. C. Percepção de estudantes do ensino fundamental quanto ao aleitamento materno e a influência da realização de palestras de educação em saúde. J. Pediatr. (Rio J.), Porto Alegre, v. 84, n. 3, p. 224-231, jun. 2008. Disponível em: $<$ http://www.scielo.br/scielo.php?script=sci_arttext\&pid=S0021$75572008000300007 \& \operatorname{lng}=\mathrm{en} \& \mathrm{nrm}=\mathrm{iso}>$. Acesso em: $30 \mathrm{dez} .2019$.

GALVÃO, M. T. G; VASCONCELOS, S. G.; PAIVA, S. S. Mulheres Doadoras de Leite Humano. Acta paul. enferm., São Paulo, v. 19, n. 2, p. 157-161, jun. 2006 . Disponível em: $<$ http://www.scielo.br/scielo.php?script=sci_arttext\&pid=S0103$21002006000200006 \& \operatorname{lng}=\mathrm{en} \& \mathrm{nrm}=\mathrm{iso}>$. Acesso em: $30 \mathrm{dez} .2019$.

NEVES, L. S.; SÁ, M. V. S.; MATTAR, M. J. G.; GALISA, M. S. Doação de leite humano: dificuldades e fatores limitantes. O Mundo da Saúde, São Paulo; v. 35, n. 2, p. 156-161, 2011. Disponível em:

<http://bvsms.saude.gov.br/bvs/artigos/doacao_leite_humano_dificuldades $\% 20 \mathrm{e} \% 20 \mathrm{fatores} \%$ 20limitantes.pdf >. Acesso em: 30 dez. 2019.

PEREIRA, R. S. V.; OLIVEIRA, M. I. C.; ANDRADE, C. L. T.; BRITO, A. S. Fatores associados ao aleitamento materno exclusivo: o papel do cuidado na atenção básica. Cad. Saúde Pública, Rio de Janeiro, v. 26, n. 12, p. 2343-2354, dez 2010. Disponível em: <https://www.scielosp.org/pdf/csp/2010.v26n12/2343-2354/pt>. Acesso em: 30 dez. 2019.

SANTOS, M. P. Extensão universitária: espaço de aprendizagem profissional e suas relações com o ensino e a pesquisa na educação superior. Revista Conexão UEPG, v. 8, n. 2, p. 154163, jul/dec. 2012. Universidade Estadual de Ponta Grossa. Ponta Grossa, Brasil. Disponível em: Disponível em: <http://www.redalyc.org/articulo.oa?id=514151728002〉. Acesso em: 30 dez. 2019.

SILVA, C. M.; PELLEGRINELLI, A. L. R.; PEREIRA, S. C. L.; PASSOS, I. R.; SANTOS, L. C. Práticas educativas segundo os "Dez passos para o sucesso do aleitamento materno" em 
um Banco de Leite Humano. Ciência \& Saúde Coletiva, vol. 22, núm. 5, p. 1661-1671, 2017. Disponível em:

<https://www.scielosp.org/article/ssm/content/raw/?resource_ssm_path=/media/assets/csc/v22 n5/1413-8123-csc-22-05-1661.pdf>. Acesso em: 30 dez. 2019.

VICTORA, C.; BAHL, R.; BARROS, A.; FRANCA, G.V.A; HORTON, S.; KRASEVEC, J.; MURCH, S.; SANKAR, M. J.; WALKER, N.; ROLLINS, N. C. Breastfeeding in the 21st Century: Epidemiology, Mechanisms and Lifelong Effect. The Lancet, v. 387, n. 10017, p. 475-490, 2016. Disponível em: <https://www.thelancet.com/journals/lancet/article/PIIS01406736(15)01024-7/fulltext>. Acesso em: 30 dez. 2019. 